\title{
Towards Non-Invasive EEG-based Arm/Hand- Control in Users with Spinal Cord Injury
}

\author{
Gernot R. Müller-Putz, Patrick Ofner, Andreas Schwarz, Joana Pereira, Andreas Pinegger, Catarina Lopes Dias, \\ Lea Hehenberger, Reinmar Kobler, Andreea Ioana Sburlea \\ Institute of Neural Engineering, BCI Lab \\ Graz University of Technology \\ Graz, Austria \\ gernot.mueller@tugraz.at
}

\begin{abstract}
Restoring the ability to reach and grasp can dramatically improve quality of life for people with cervical spinal cord injury (SCI). The main challenge in restoring independent reaching and grasping in patients is to develop assistive technologies with intuitive and non-invasive user interfaces. We believe that this challenge can be met by directly translating movement-related brain activity into control signals. During the last decade, we have conducted research on EEG-based braincomputer interfaces (BCIs) for the decoding of movement parameters, such as trajectories and targets. Although our findings are promising, the control is still unnatural. Therefore, we surmise that natural and intuitive control of neuroprostheses could be achieved by developing a novel control framework that incorporates detection of goal directed movement intention, movement decoding, identifying the type of grasp, error potentials detection and delivery of feedback.
\end{abstract}

Keywords - EEG, brain-computer interface, natural movement control, spinal cord injury

\section{INTRODUCTION}

Grasping an apple, brushing teeth, or simply playing around with the fingers is not possible for people who received a cervical spinal cord injury. Depending on the damaged area, motor commands from the brain to the periphery (efferent) or sensory signals from the periphery to the brain (afferent) cannot bypass the interruption in the spinal cord. One approach to provide help for this group of people is to detect the intention of their movement and to reproduce the movement of their paralyzed arm/hand with the help of a motor neuroprosthesis or artificial arm.

In the past we have shown that it is possible to combine brain-computer interfaces with motor neuroprostheses [1]. We used classical motor imagery of sustained feet or hand movement in a single class BCI to switch between predefined grasp phases [2], [3]. Later, we applied our methodology for elbow movements in healthy subjects and in end-users with SCI [4], [5], [6].

The introduction of hybrid BCIs [7], [8], known as the fusion of a BCI with a second input modality (e.g., shouldercontrolled joystick), allowed the user to benefit from the advantages of both technologies [9]. Various studies showed the benefits of the hybrid BCI concept over classical BCI [10], [11]. However, these strategies have the drawback of not reflecting the natural way of planning a movement. To achieve an ecological and intuitive control, the movements decoded by the $\mathrm{BCI}$ need to be closely related to the user's intention.

\section{Methods \& Results}

In the following section we describe the results of first experiments which are the basis of our research towards a natural neuroprosthesis control based on non-invasive EEG signals.

\section{A. Goal directed movement detection}

Movement-related cortical potentials (MRCPs) are EEG neural correlates of movement which have been used for movement intention detection and are known to be modulated by several movement-related parameters [12]. We study MRCPs during goal-directed movement tasks to assess whether goal-directed strategies should be preferred in the context of BCI control [13]. Our first results on healthy subjects suggest that movement detection is significantly improved in goaldirected movement execution when compared to the same task (i.e. same movement kinematics) without a specific target. These differences are particularly visible during movement planning. Such performance improvements can lead to a reliable and natural response of the BCI-controlled system to the user's intention. By estimating the brain sources of the discriminative spatial patterns, we further concluded that the differences between the two motor tasks were associated to motor areas and the posterior parietal lobule.

\section{B. Movement decoding from executed and imagined arm movements}

We showed in [14] the decoding of executed hand movements with a linear decoding model from low-frequency EEG signals. The Pearson correlation coefficients between the decoded and measured hand positions were $0.70,0.78$ and 
0.62 for the $\mathrm{x}, \mathrm{y}$, and $\mathrm{z}$ dimension, respectively. Based on this decoder, we discriminated between imagined horizontal and vertical hand movements with astatistically significant classification accuracy of $64 \%$ [15].

\section{Decoding of movement target/direction}

In a preliminary study employing a center-out reaching task to 4 targets displayed on a computer screen, all 3 subjects reached a statistically significantly classification accuracy of $42 \%, 47 \%$ and $73 \%$ [16].

\section{Single joint movement classification}

Based on EEG signals from 0.3 to $3 \mathrm{~Hz}$, we found 6 different upper-limb movements to be discriminable with a classification accuracy of $37 \%$ in a group of 15 healthy subjects [17]. The classifier sources originated mainly on premotor and primary motor areas.

\section{E. Classification of different types of grasp}

Despite the high number of degrees of freedom of the human hand, most actions of daily life can be executed using just palmar, pinch or lateral grasp [18]. Therefore we conducted an EEG-based study in 15 healthy users to find out whether these grasps can be discriminated from each other and from a no-movement condition. Using a cue-guided paradigm, we recorded 72 trials of executed reach and grasp actions for each condition. Our results show that time-domain features located in the low frequency range $(0.3-3 \mathrm{~Hz})$ provide sufficient information for meaningful classification [19].

\section{DiscussiON}

With the presented work we laid the basis for a series of several studies in these specific areas, with the final aim of developing a new decoder system. Using the new system end users with SCI will be able to control neuroprosthethic arm movements in 3D space with the goal to grasp and manipulate objects. However, several aspects need to be addressed further.

When the user controls a robotic arm or neuroprosthesis, visual feedback is inherent. Without experimental restrictions, the eyes would track points of interest (e.g. end-effector and target object). It is therefore imperative to remove the resulting electroocular artifacts in order to obtain reliable control based on EEG brain activity.

In addition, we aim to incorporate a classifier of errorrelated potentials, as a backup system. When the main classifier fails, and the prosthesis's movement does not correspond to the user's intent, the user will perceive this action as an error and therefore generate an error-related potential. By detecting errorrelated potentials during the continuous movement of the prosthesis, its trajectory can be automatically corrected or interrupted, allowing a smoother and faster interaction between the user and the prosthesis.
Intuitive control of a neuroprosthesis necessitates artificial feedback to compensate for the absence of the comprehensive feedback provided to a healthy person via intact afferent pathways, such as the sense of touch, proprioception or grasp force.

\section{CONCLUSION}

In conclusion, our findings highlight the growing interest for non-invasive EEG-based BCI and its potential for the recovery of upper limb lost functions in individuals with spinal cord injury. The results of our studies based on the detection of goal directed movement intention, movement decoding, identifying the type of grasp, error potentials detection and delivery of feedback, show a promising perspective towards achieving a natural and intuitive control of neuroprostheses.

\section{ACKNOWLEDGMENT}

This work was supported by the Horizon 2020 RIA project MoreGrasp (643955) and the ERC Consolidator Grant Feel Your Reach (681231).

\section{REFERENCES}

[1] Müller-Putz, Gernot R., et al. "Brain-computer interfaces for control of neuroprostheses: from synchronous to asynchronous mode of operation/Brain-Computer Interfaces zur Steuerung von Neuroprothesen: von der synchronen zur asynchronen Funktionsweise." Biomedizinische Technik 51.2 (2006): 57-63

[2] Pfurtscheller, Gert, et al. "'Thought'-control of functional electrical stimulation to restore hand grasp in a patient with tetraplegia." Neuroscience letters 351.1 (2003): 33-36.

[3] Müller-Putz, Gernot R., et al. "EEG-based neuroprosthesis control: a step towards clinical practice." Neuroscience letters 382.1 (2005): 169174

[4] Mueller-Putz, Gernot, et al. "Temporal coding of brain patterns for direct limb control in humans." Frontiers in neuroscience 4 (2010): 34.

[5] Wu, Zhaohui, et al. "The convergence of machine and biological intelligence." IEEE Intelligent Systems 28.5 (2013): 28-43.

[6] Rupp, R., et al. "Think2grasp-BCI-controlled neuroprosthesis for the upper extremity." Biomedical Engineering/Biomedizinische Technik (2013).

[7] Pfurtscheller, Gert, et al. "The hybrid BCI." Frontiers in neuroscience 4 (2010): 3 .

[8] Müller-Putz, Gernot R., et al. "Tools for brain-computer interaction: a general concept for a hybrid BCI." Frontiers in neuroinformatics 5 (2011): 30

[9] Rohm, Martin, et al. "Hybrid brain-computer interfaces and hybrid neuroprostheses for restoration of upper limb functions in individuals with high-level spinal cord injury." Artificial intelligence in medicine 59.2 (2013): 133-142

[10] Müller-Putz, Gernot, et al. "Towards noninvasive hybrid brain-computer interfaces: framework, practice, clinical application, and beyond." Proceedings of the IEEE 103.6 (2015): 926-943.

[11] Rupp, Rüdiger, et al. "Functional rehabilitation of the paralyzed upper extremity after spinal cord injury by noninvasive hybrid neuroprostheses." Proceedings of the IEEE 103.6 (2015): 954-968.

[12] Jochumsen, Mads, et al. "Comparison of spatial filters and features for the detection and classification of movement-related cortical potentials 
in healthy individuals and stroke patients." Journal of neural engineering 12.5 (2015): 056003

[13] Pereira, Joana, Patrick Ofner, and Gernot R. Müller-Putz. "Goal-directed or aimless? EEG differences during the preparation of a reach-and-touch task." 2015 37th Annual International Conference of the IEEE Engineering in Medicine and Biology Society (EMBC). IEEE, 2015.

[14] Ofner, Patrick, and Gernot R. Müller-Putz. "Decoding of velocities and positions of 3D arm movement from EEG." 2012 Annual International Conference of the IEEE Engineering in Medicine and Biology Society. IEEE, 2012.

[15] Ofner, Patrick, and Gernot R. Müller-Putz. "Using a noninvasive decoding method to classify rhythmic movement imaginations of the arm in two planes." IEEE Transactions on Biomedical Engineering 62.3 (2015): 972-981.
[16] Ofner, Patrick, and Gernot R. Müller-Putz. "Movement target decoding from EEG and the corresponding discriminative sources: A preliminary study." 2015 37th Annual International Conference of the IEEE Engineering in Medicine and Biology Society (EMBC). IEEE, 2015.

[17] Ofner, Patrick et al. "Movements of the same upper limb can be classified from low-frequency time-domain EEG signals", Proceedings of the 6th International Brain-Computer Interface Meeting,2016.

[18] Popović, Dejan B., and Thomas Sinkjær. "Central nervous system lesions leading to disability." J Aut Control 18.2 (2008): 13-24.

[19] Müller-Putz, G. R., et al. "From classic motor imagery to complex movement intention decoding: The noninvasive Graz-BCI approach." Progress in Brain Research (2016). 